\title{
Global Property in a Delayed Periodic Predator-Prey Model with Stage-Structure in Prey and Density-Independence in Predator
}

\author{
Xiaolin Fan, ${ }^{1,2}$ Zhidong Teng, ${ }^{1}$ and Haijun Jiang ${ }^{1}$ \\ ${ }^{1}$ College of Mathematics and System Sciences, Xinjiang University, Urumqi, Xinjiang 830046, China \\ ${ }^{2}$ Department of Basic Education, Xinjiang Institute of Engineering, Urumqi, Xinjiang 830091, China \\ Correspondence should be addressed to Zhidong Teng; zhidong1960@163.com
}

Received 14 December 2013; Accepted 7 February 2014; Published 16 March 2014

Academic Editor: Kaifa Wang

Copyright (c) 2014 Xiaolin Fan et al. This is an open access article distributed under the Creative Commons Attribution License, which permits unrestricted use, distribution, and reproduction in any medium, provided the original work is properly cited.

\begin{abstract}
We study the global property in a delayed periodic predator-prey model with stage-structure in prey and density-independence in predator. The sufficient conditions on the ultimate boundedness of all positive solutions are obtained, and the sufficient conditions of the integrable form for the permanence and extinction are further established, respectively. Some well-known results on the predator density-dependency are improved and extended to the predator density-independent cases. The theoretical results are confirmed by the special examples and the numerical simulations.
\end{abstract}

\section{Introduction}

There are many different kinds of two-species predatorprey dynamical models in mathematical ecology. Particularly, two-species predator-prey model with stage-structure have been extensively studied by a large number of papers, see [1-5] and the reference cited therein. The main research topics include the persistence, permanence and extinction of species, the existence and the global asymptotic properties of positive periodic solutions in periodic case, and the global stability of models in general nonautonomous cases.

In [2], Cui and Song studied a periodic predator-prey system with stage-structure. They provided a sufficient and necessary condition to guarantee the permanence of species for the system. In [3], Cui and Takeuchi studied a periodic predator-prey system with stage-structure with function response. They provided a sufficient and necessary condition to guarantee the permanence of species for the system with infinite delay. Some known results are extended to the delay case.

So far, from these done works on the predator-prey model with stage-structure, the authors always assume that the predator is strictly density-dependent, which is much identical with the real biological background. On the other hand, the effect of periodically varying environment plays an important role in the permanence and extinction of species for the system (e.g., seasonal effects of climate, food supply, mating habits, hunting or harvesting seasons, etc.). Thus, the assumptions of periodicity of the parameters and system with time delay are effective ways to characterize and investigate population systems. Owing to many natural and man-made factors such as the low birth rate, high death rate, decreasing habitats, and the hunting of human beings, and the worse ecological system, some predator species become rare and even liable to extinction. For these predator species, we can ignore the effect of density-dependency. Up to now, there are some works on such investigation for the situation of predator density-independence. The authors always assume that the density of predator is proportional to the predation rate, the conversion rate of the immature prey biomass into predator biomass, and the death rate of predator. Predator densityindependece is reasonable to the real ecosystem.

To our knowledge, few scholars consider the delayed periodic predator-prey models with stage-structure in prey 
and density-independence in predator. In this paper, we consider the following system:

$$
\begin{gathered}
\frac{d x_{1}(t)}{d t}=a(t) x_{2}(t)-b(t) x_{1}(t)-d(t) x_{1}^{2}(t) \\
\quad-p(t) \phi\left(x_{1}(t)\right) \int_{-h}^{0} k_{12}(s) y(t+s) d s, \\
\frac{d x_{2}(t)}{d t}=c(t) x_{1}(t)-f(t) x_{2}^{2}(t), \\
\frac{d y(t)}{d t}=y(t)\left[-g(t)+h(t) \int_{-h}^{0} k_{21}(s) \phi\left(x_{1}(t+s)\right) d s\right] .
\end{gathered}
$$

Our purpose in this paper is to establish sufficient conditions of integrable form for the permanence and extinction of species for system (1). By using the analysis method, the comparison theorem of cooperative system, and the theory of the persistence of dynamical systems, the integral form criteria on the ultimate boundedness, permanence, and extinction are established. The method used in this paper is motivated by the works on the permanence and extinction for periodic predator-prey systems in patchy environment given by Teng and Chen in [5].

The organization of this paper is as follows. In the next section, the basic assumptions for system (1), some notations, and lemmas which will be used in the later sections are introduced as the preliminaries. In Section 3, the main results of this paper are stated. In Section 4, the proofs of the main theorems are given. In Section 5, the theoretical results are confirmed by some special examples and the numerical simulations. Finally, a conclusion is given in Section 6.

\section{Preliminaries}

In system $(1), x_{i}(t)(i=1,2)$ represent the population density of the infancy prey and maturity prey at time $t$, respectively, and $y(t)$ represents the population density of predator species at time $t$ which only prey on infancy prey $x_{1}(t)$. Functions $a(t), b(t), c(t), d(t), f(t), g(t), h(t)$, and $p(t)$ are periodic and continuous defined on $R_{+}=[0, \infty)$ with common period $\omega>0$, and $a(t), b(t), c(t), d(t)$, and $f(t)$ are also positive, where $a(t), b(t)$, and $d(t)$ denote the birthrate, mortality, and density restriction of infancy prey $x_{1}(t)$ at time $t$, respectively, $c(t)$ denotes the transformation from the infancy prey $x_{1}(t)$ to the maturity prey $x_{2}(t)$ at time $t, f(t)$ denotes the mortality and density restriction of maturity prey $x_{2}(t)$ at time $t, p(t)$ denotes the predation rate in which the predator $y(t)$ captures the infancy prey $x_{1}(t)$ at time $t, g(t)$ is the mortality of predator $y(t)$ at time $t$, and $h(t)$ denotes the transformation from the infancy prey $x_{1}(t)$ to the predator $y(t)$ by the assimilation. Functions $k_{i j}(s)(i, j=1,2)$ are nonnegative and integrable on $[-h, 0]$ and $\int_{-h}^{0} k_{i j}(s) d s=1$. Function $\phi\left(x_{1}\right)$, the number of the prey consumed per predator in unit time, is called the predator functional response. In this paper, we always assume that $\phi\left(x_{1}\right)$ is continuous differentiable function and $\phi(0)=0$.
We define set $C_{+}$as follows:

$$
\begin{aligned}
& C_{+}=\left\{\psi=\left(\psi_{1}, \psi_{2}, \psi_{3}\right): \psi_{i}(s)\right. \text { is nonnegative } \\
&\text { continous for } \left.s \in[-h, 0], \psi_{i}(0)>0, i=1,2,3\right\} .
\end{aligned}
$$

For any $\psi \in C_{+}$, the norm is defined by $\|\psi\|=$ $\sup _{-h \leq \theta \leq 0}|\psi(\theta)|$. Motivated by the biological background of system (1), in this paper, we always assume that the solutions of system (1) satisfy the following initial conditions:

$$
\begin{gathered}
x_{1}(s)=\psi_{1}(s), \quad x_{2}(s)=\psi_{2}(s), \\
y(s)=\psi_{3}(s), \quad-h \leqslant s \leqslant 0,
\end{gathered}
$$

where $\psi=\left(\psi_{1}, \psi_{2}, \psi_{3}\right) \in C_{+}$. It is easy to prove that the right functional of system (1) is continuous and satisfies a local Lipschitz condition with respect to $\psi=\left(\psi_{1}, \psi_{2}, \psi_{3}\right) \in C_{+}$. Therefore, by the fundamental theory of functional differential equations (see [6-8]), for any $\psi=\left(\psi_{1}, \psi_{2}, \psi_{3}\right) \in C_{+}$, system (1) has a unique solution $\left(x_{1}(t, \psi), x_{2}(t, \psi), y(t, \psi)\right)$ satisfying initial condition (3). It is also easy to prove that the solution $\left(x_{1}(t, \psi), x_{2}(t, \psi), y(t, \psi)\right)$ is positive; that is, $x_{i}(t, \psi)>0(i=1,2)$ and $y(t, \psi)>0$ in its maximal interval of existence. In this paper, such a solution of system (1) is called a positive solution.

Let $f(t)$ be a $\omega$-periodic continuous function defined on $R_{+}$, we define

$$
\begin{gathered}
A_{\omega}(f)=\omega^{-1} \int_{0}^{\omega} f(t) d t, \quad f^{m}=\max _{t \in R^{+}} f(t), \\
f^{l}=\min _{t \in R^{+}} f(t) .
\end{gathered}
$$

Consider the following differential equations system:

$$
\begin{aligned}
& \frac{d u(t)}{d t}=\alpha(t) v(t)-\beta(t) u(t)-\gamma(t) u^{2}(t), \\
& \frac{d v(t)}{d t}=\delta(t) u(t)-\eta(t) v^{2}(t),
\end{aligned}
$$

where functions $\alpha(t), \beta(t), \gamma(t), \delta(t)$, and $\eta(t)$ are positive periodic and continuous defined on $R_{+}$with common period $\omega>0$. We have the following result.

Lemma 1 (see [9]). System (5) has a positive w-periodic solution $\left(u^{*}(t), v^{*}(t)\right)$ which is globally asymptotically stable.

Remark 2. Directly from system (5), we can obtain that when we increase coefficients $\alpha(t)$ and $\delta(t)$, or decrease coefficients $\beta(t), \gamma(t)$, and $\eta(t)$, then $u^{*}(t)$ and $v^{*}(t)$ will largen. Otherwise, $u^{*}(t)$ and $v^{*}(t)$ will decrease.

When the predator species $y(t)=0$ in system (1), we obtain the following subsystem of system (1):

$$
\begin{aligned}
& \frac{d x_{1}(t)}{d t}=a(t) x_{2}(t)-b(t) x_{1}(t)-d(t) x_{1}^{2}(t), \\
& \frac{d x_{2}(t)}{d t}=c(t) x_{1}(t)-f(t) x_{2}^{2}(t)
\end{aligned}
$$


It is clear that the solution $\left(x_{1}(t), x_{2}(t)\right)$ of system (6) with initial value $\left(x_{1}(0), x_{2}(0)\right)>0$ is positive for all $t>0$. We further have the following result as a corollary of Lemma 1.

Corollary 3. System (6) has a positive w-periodic solution $\left(x_{1}^{*}(t), x_{2}^{*}(t)\right)$ which is globally asymptotically stable.

Remark 4. As a direct consequence of Corollary 3, we see that system (1) has a predator extinction periodic solution $\left(x_{1}^{*}(t), x_{2}^{*}(t), 0\right)$.

Remark 5. Obviously, from Remark 2, by increasing coefficients $a(t)$ and $c(t)$, or decreasing coefficients $b(t), d(t)$, and $f(t)$, we can see that $x_{1}^{*}(t)$ and $x_{2}^{*}(t)$ will largen. Otherwise, $x_{1}^{*}(t)$ and $x_{2}^{*}(t)$ will decrease.

For system (1), we introduce the following basic assumptions:

$$
\begin{aligned}
& \left(A_{1}\right) A_{\omega}(g)>0 \\
& \left(A_{2}\right) p^{l}>0 \text { and } h^{l} \geq 0 \\
& \left(A_{3}\right) \phi^{\prime}\left(x_{1}\right) \geqslant 0 \text { for all } 0 \leqslant x_{1} \leqslant \max _{t \in(0, \omega]} x_{1}^{*}(t) \text {, and } \phi(0)= \\
& \quad 0 .
\end{aligned}
$$

Let $X$ be a complete metric space with metric $d$. Suppose that $f: X \rightarrow X$ is a continuous map. For any $x \in X$, we denote $f^{n}(x)=f\left(f^{n-1}(x)\right)$ for any integer $n>1$ and $f^{1}(x)=$ $f(x)$. $f$ is said to be compact in $X$, if for any bounded set $H \subset X$ set $f(H)=\{f(x): x \in H\}$ is precompact in $X . f$ is said to be point dissipative if there is a bounded set $B_{0} \subset X$ such that for any $x \in X$. Consider

$$
\lim _{n \rightarrow \infty} d\left(f^{n}(x), B_{0}\right)=0 .
$$

For any $x_{0} \in X$, the positive semiorbit through $x_{0}$ is defined by $\gamma^{+}\left(x_{0}\right)=\left\{x_{n}=f^{n}\left(x_{0}\right): n=1,2, \ldots\right\}$, the negative semiorbit through $x_{0}$ is defined as a sequence $\gamma^{-}\left(x_{0}\right)=\left\{x_{k}\right\}$ satisfying $f\left(x_{k-1}\right)=x_{k}$ for integers $k \leqslant 0$, and its $\omega$-limit set is $\omega\left(x_{0}\right)=\left\{y \in X\right.$; there is a time sequence $n_{k} \rightarrow \infty$ such that $\lim _{k \rightarrow \infty} f\left(x_{n_{k}}\right)=y$ \} and its $\alpha$-limit set is $\alpha\left(x_{0}\right)=$ $\left\{y \in X\right.$; there is a time sequence $n_{k} \rightarrow-\infty$ such that $\left.\lim _{k \rightarrow \infty} f\left(x_{n_{k}}\right)=y\right\}$.

A nonempty set $A \subset X$ is said to be invariant if $f(A) \subseteq$ A. A nonempty invariant set $M$ of $X$ is called to be isolated in $X$, if it is the maximal invariant set in a neighborhood of itself. For a nonempty set $M$ of $X$, set $W^{s}(M):=\{x \in X$ : $\left.\lim _{n \rightarrow \infty} d\left(f^{n}(x), M\right)=0\right\}$ is called the stable set of $M$.

Let $A$ and $B$ be two isolated invariant sets; set $A$ is said to be chained to set $B$, written as $A \rightarrow B$, if there exists a full orbit though some $x \notin A \cup B$ such that $\omega(x) \subset B$ and $\alpha(x) \subset$ $A$. A finite sequence $\left\{M_{1}, \ldots, M_{k}\right\}$ of isolated invariant sets is called a chain, if $M_{1} \rightarrow M_{2} \rightarrow \cdots \rightarrow M_{k}$, and if $M_{k}=M_{1}$, the chain is called a cycle.

Let $X^{0}$ and $\partial X^{0}$ be nonempty open set and nonempty closed set of $X$, respectively, and satisfying $X^{0} \cap \partial X^{0}=\emptyset$. We denote

$$
M_{\partial}=\left\{x \in \partial X^{0}: f^{n}(x) \in \partial X^{0}, \forall n \geq 0\right\} .
$$

Lemma 6. Let $f: X \rightarrow X$ be a continuous map. Assume that the following conditions hold:
$\left(C_{1}\right) f$ is compact and point dissipative, and $f\left(X^{0}\right) \subseteq X^{0}$;

$\left(C_{2}\right)$ there exists a finite sequence $\mathscr{M}=\left\{M_{1}, \ldots, M_{k}\right\}$ of compact and isolated invariant sets such that

(a) $M_{i} \cap M_{j}=\emptyset$ for any $i, j=1,2, \ldots, k$ and $i \neq j$;

(b) $\Omega\left(M_{\partial}\right):=\cup\left\{\omega(x): x \in M_{\partial}\right\} \subset \cup_{i=1}^{k} M_{i}$;

(c) no subset of $\mathscr{M}$ forms a cycle in $\partial X^{0}$;

(d) $W^{s}\left(M_{i}\right) \cap X^{0}=\emptyset$ for each $1 \leq i \leq k$.

Then $f$ is uniformly persistent with respect to $\left(X^{0}, \partial X^{0}\right)$; that is, there exists a constant $\eta>0$ such that $\liminf _{n \rightarrow \infty} d\left(f^{n}(x), \partial X^{0}\right) \geqslant \eta$ for all $x \in X^{0}$.

Lemma 6 can be obtained from Theorem 1.1.3, Theorem 1.3.1, Remark 1.3.1, and Theorem 1.3.3 given by Zhao in [10].

\section{Main Results}

Firstly, concerning the persistence and permanence of species for system (1), we have the following general result.

Theorem 7. Suppose that $\left(A_{1}\right)-\left(A_{3}\right)$ hold. Then there exists a positive constant $M>0$ such that

$$
\begin{gathered}
\lim \sup _{t \rightarrow \infty} x_{i}(t) \leq M \quad(i=1,2), \\
\lim \sup _{t \rightarrow \infty} y(t) \leq M
\end{gathered}
$$

for any positive solution $\left(x_{1}(t), x_{2}(t), y(t)\right)$ of system (1).

Remark 8. Let us see the biological meaning of Theorem 7. In fact, if the predator species is not ultimately bounded, then the population density of predator species will expand unlimitedly. Since the predation rate of predator species for prey species is strictly positive (i.e., $p^{l}>0$ in assumption $\left(A_{2}\right)$ ), the prey species will become extinct because of the massive preying by the predator species. Since the survival of predator is absolutely dependent on the prey species, as an opposite result, the predator species will become extinct too.

However, if the predation rate $p(t)$ of the predator species is not strictly positive, that is, $p^{l}=0$, then it cannot lead to extinction when the population density of predator species expands unlimitedly. Therefore, an important open question is whether we can still obtain the boundedness of predator species which is density-independent when $p^{l}=0$.

Theorem 9. Suppose that $\left(A_{1}\right)-\left(A_{3}\right)$ hold. If

$$
A_{\omega}\left[-g(t)+h(t) \int_{-h}^{0} k_{21}(s) \phi\left(x_{1}^{*}(t+s)\right) d s\right]>0
$$

where $\left(x_{1}^{*}(t), x_{2}^{*}(t)\right)$ is the positive $\omega$-periodic solution of system (6), then system (1) is uniformly persistent. That is, there exists a positive constant $\epsilon$, such that any solution $\left(x_{1}(t), x_{2}(t), y(t)\right)$ of system (1) with initial condition (3) satisfies

$$
\liminf _{t \rightarrow \infty}\left(x_{1}(t), x_{2}(t), y(t)\right) \geqslant(\epsilon, \epsilon, \epsilon) .
$$


Remark 10. Theorem 9 shows that if we guarantee that $\left(A_{1}\right)-\left(A_{3}\right)$ hold, then the prey species must be permanent. In fact, if the prey species $\left(x_{1}(t), x_{2}(t)\right)$ is not permanent, then it may be extinct, as a result the predator species $y(t)$ will be extinct too because its survival is absolutely dependent on $x_{1}(t)$. However, when predator species $y(t)$ become extinct, prey species $\left(x_{1}(t), x_{2}(t)\right)$ will not turn to extinction, because $\left(A_{1}\right)$ shows that $x_{1}(t)$ has a total positive average growth rate.

Remark 11. From Lemma 1, we know that, when there is no predator species $y(t)$, the prey species $\left(x_{1}(t), x_{2}(t)\right)$ will approach a positive periodic solution stable state $\left(x_{1}^{*}(t), x_{2}^{*}(t)\right)$. When there is predator species $y(t)$, Theorem 9 shows that if the positive periodic stable state $\left(x_{1}^{*}(t), x_{2}^{*}(t)\right)$ of prey species $\left(x_{1}(t), x_{2}(t)\right)$ can guarantee that predator species $y(t)$ obtain a positive total average growth rate, that is, condition (10), then predator species $y(t)$ will be permanent.

Theorem 12. Suppose that $\left(A_{1}\right)-\left(A_{3}\right)$ hold. If

$$
A_{\omega}\left[-g(t)+h(t) \int_{-h}^{0} k_{21}(s) \phi\left(x_{1}^{*}(t+s)\right) d s\right]<0,
$$

then for any positive solution $\left(x_{1}(t), x_{2}(t), y(t)\right)$ of system (1), $x_{i}(t) \rightarrow x_{i}^{*}(t)(i=1,2)$ and $y(t) \rightarrow 0$, as $t \rightarrow \infty$.

Remark 13. Theorem 12 shows that when the prey species $\left(x_{1}(t), x_{2}(t)\right)$ approach a positive periodic solution stable state $\left(x_{1}^{*}(t), x_{2}^{*}(t)\right)$, the predator species $y(t)$ can only obtain a negative total average growth rate, that is, condition (12), then $y(t)$ will be extinct.

Lastly, from Theorems 9 and 7 given by Teng and Chen in [11] on the existence of positive periodic solutions for general Kolmogorov systems with bounded delays, we have the following result.

Corollary 14. Suppose that $\left(A_{1}\right)-\left(A_{3}\right)$ hold. If

$$
A_{\omega}\left[-g(t)+h(t) \int_{-h}^{0} k_{21}(s) \phi\left(x_{1}^{*}(t+s)\right) d s\right]>0,
$$

then system (1) has at least a positive w-periodic solution.

Remark 15. In this paper we obtain the existence of the positive periodic solutions for system (1) under the assumption that all parameters are with common periodicity. However, considering all parameters fluctuating in time with the same period is unrealistic, because it will be more realistic if we allow time fluctuations with different period or even nonperiod with some almost periodic environment, which will be more identical with the sound ecosystem. Therefore, there is a very important open question that is whether the same result given in Lemma 1 will be true under the assumption that the parameter in system (1) is almost periodic.

Remark 16. From Remark 5 we know that by increasing coefficients $a(t)$ and $c(t)$ or decreasing coefficients $b(t), d(t)$, and $f(t)$, then $x_{1}^{*}(t)$ and $x_{2}^{*}(t)$ will largen. This shows that by increasing coefficients $a(t)$ and $c(t)$ or decreasing coefficients $b(t), d(t)$, and $f(t)$, we can get that

$$
A_{\omega}\left[-g(t)+h(t) \int_{-h}^{0} k_{21}(s) \phi\left(x_{1}^{*}(t+s)\right) d s\right]
$$

increases. Thus, condition (12) can be changed to condition (10). Therefore, from Theorems 9 and 12, we obtain that predator $y(t)$ will become into the permanence from the quondam extinction. This shows that the stage-structure in the prey (i.e., the birthrate, mortality, density restriction of infancy prey, the transformation from the infancy prey to the maturity prey, and the mortality and density restriction of maturity prey) will bring the effect for the permanence and extinction of the predator.

Remark 17. System (1) is a pure delay system with respect to $y(t)$. We cannot use the variable without time delay to control the variable with time delay. This shows that it is very difficult to get the global attractivity of system (1). We will discuss this problem in the future.

Remark 18. An important open question is that what results will be obtained with the condition

$$
A_{\omega}\left[-g(t)+h(t) \int_{-h}^{0} k_{21}(s) \phi\left(x_{1}^{*}(t+s)\right) d s\right]=0 .
$$

Is it the permanence of system (1) or the extinction of predator $y(t)$ ?

When system (1) degenerates into the nondelayed system of ordinary differential equations, that is, in system (1) $k_{i j} \equiv$ $0(i, j=1,2)$, then we have

$$
\begin{aligned}
& \frac{d x_{1}(t)}{d t}=a(t) x_{2}(t)-b(t) x_{1}(t) \\
& \quad-d(t) x_{1}^{2}(t)-p(t) \varphi\left(x_{1}(t)\right) y(t), \\
& \frac{d x_{2}(t)}{d t}=c(t) x_{1}(t)-f(t) x_{2}^{2}(t), \\
& \frac{d y(t)}{d t}=y(t)\left[-g(t)+h(t) \varphi\left(x_{1}(t)\right)\right] ;
\end{aligned}
$$

we can see that the above assumptions for system (16) will have the following forms:

$$
\begin{aligned}
& \left(A_{1}^{*}\right) A_{\omega}(g)>0 \\
& \left(A_{2}^{*}\right) p^{l}>0 \text { and } h^{l} \geq 0 . \\
& \left(A_{3}^{*}\right) \varphi^{\prime}\left(x_{1}\right) \geq 0 \text { and } \varphi(0)=0 \text { for } 0 \leq x_{1} \leq \max _{t \in(0, \omega]} x_{1}^{*}(t) .
\end{aligned}
$$

Therefore, as special cases of Theorems $7-12$ we have the following results for system (16).

Corollary 19. Suppose that $\left(A_{1}^{*}\right)-\left(A_{3}^{*}\right)$ hold. Then there exists a positive constant $M>0$ such that

$$
\begin{gathered}
\lim \sup _{t \rightarrow \infty} x_{i}(t) \leq M \quad(i=1,2), \\
\lim \sup _{t \rightarrow \infty} y(t) \leq M,
\end{gathered}
$$

for any positive solution $\left(x_{1}(t), x_{2}(t), y(t)\right)$ of system (16). 
Corollary 20. Suppose that $\left(A_{1}^{*}\right)-\left(A_{3}^{*}\right)$ hold. If

$$
A_{\omega}\left[-g(t)+h(t) \varphi\left(x_{1}^{*}(t)\right)\right]>0,
$$

then system (16) is uniformly persistent, where $\left(x_{1}^{*}(t), x_{2}^{*}(t)\right)$ is the positive $\omega$-periodic solution of system (6).

Corollary 21. Suppose that $\left(A_{1}^{*}\right)-\left(A_{3}^{*}\right)$ hold. If

$$
A_{\omega}\left[-g(t)+h(t) \varphi\left(x_{1}^{*}(t)\right)\right]<0,
$$

then for any positive solution $\left(\left(x_{1}(t), x_{2}(t), y(t)\right)\right.$ of system (16), $x_{i}(t) \rightarrow x_{i}^{*}(t)(i=1,2)$ and $y(t) \rightarrow 0$ as $t \rightarrow \infty$.

\section{Proof of Theorems}

Proof of Theorem 7. For any positive solution $\left(x_{1}(t), x_{2}(t)\right.$, $y(t))$ of system (1), we have

$$
\begin{aligned}
& \frac{d x_{1}(t)}{d t} \leq a(t) x_{2}(t)-b(t) x_{1}(t)-d(t) x_{1}^{2}(t), \\
& \frac{d x_{2}(t)}{d t}=c(t) x_{1}(t)-f(t) x_{2}^{2}(t) .
\end{aligned}
$$

By the vector comparison theorem (see $[12,13])$ and Corollary 3 , we can obtain that for any $\varepsilon>0$ there is a $T_{1}>0$ such that

$$
x_{i}(t) \leq x_{i}^{*}(t)+\varepsilon, \quad i=1,2, \forall t \geq T_{1} .
$$

This leads to

$$
\limsup _{t \rightarrow \infty} x_{i}(t) \leq M_{1}, \quad i=1,2,
$$

where $M_{1}=\max \left\{x_{i}^{*}(t): i=1,2, t \in[0, \omega]\right\}$.

Next, we prove that there exists a positive constant $M_{2}>0$ such that

$$
\limsup _{t \rightarrow \infty} y(t) \leq M_{2}
$$

And, from $\left(A_{1}\right)$ and $\left(A_{2}\right)$, we can choose positive constants $M_{0}>M_{1}$ and $0<\epsilon_{0}<M_{1}$ such that

$$
\begin{gathered}
M_{1} a^{m}-p^{l} M_{0} \phi\left(\epsilon_{0}\right)<-\epsilon_{0}, \\
A_{\omega}\left[-g(t)+h(t) \phi\left(\epsilon_{0}\right)\right]<-\epsilon_{0} .
\end{gathered}
$$

We firstly prove that

$$
\liminf _{t \rightarrow \infty} y(t) \leq M_{0}
$$

Otherwise, there exists a positive constant $T_{2}>T_{1}$ such that $y(t)>M_{0}$ for all $t \geq T_{2}$. If $x_{1}(t) \geq \epsilon_{0}$ for all $t \geq T_{2}+h$, then, for any $t \geq T_{2}+h$, we have by (24)

$$
\begin{aligned}
\frac{d x_{1}(t)}{d t} & \leq a(t) x_{2}(t)-p(t) \phi\left(x_{1}(t)\right) \int_{-h}^{0} k_{12}(s) y(t+s) d s \\
& <a(t) x_{2}(t)-p(t) \phi\left(x_{1}(t)\right) M_{0} \\
& <M_{1} a^{m}-p^{l} M_{0} \phi\left(\epsilon_{0}\right)<-\epsilon_{0} .
\end{aligned}
$$

Integrating (27) from $T_{2}+h$ to $t$ we have

$$
x_{1}(t) \leq x_{1}\left(T_{2}+h\right)-\epsilon_{0}\left(t-T_{2}-h\right),
$$

which implies to $x_{1}(t) \rightarrow-\infty$ as $t \rightarrow \infty$. This leads a contradiction. Therefore, there is a $t_{1}>T_{2}+h$ such that $x_{1}\left(t_{1}\right)<$ $\epsilon_{0}$. Now, we prove that $x_{1}(t)<\epsilon_{0}$ for all $t \geq t_{1}$. Otherwise, there exists a $t_{2}>t_{1}$ such that $x_{1}\left(t_{2}\right)=\epsilon_{0}$ and $x_{1}(t)<\epsilon_{0}$ for all $t \in\left(t_{1}, t_{2}\right)$. Then, we have $d x_{1}\left(t_{2}\right) / d t \geq 0$. On the other hand, from the first equation of system (1), a similar calculation as in (27), we have

$$
\frac{d x_{1}\left(t_{2}\right)}{d t} \leq M_{1} a^{m}-p^{l} M_{0} \phi\left(\epsilon_{0}\right) \leq-\epsilon_{0}
$$

which leads to a contradiction. Thus, $x_{1}(t)<\epsilon_{0}$ for all $t \geq t_{1}$. For any $t \geq t_{1}+h$, we choose an integer $p_{t}>0$ such that $t \in\left[t_{1}+h+p_{t} \omega, t_{1}+h+\left(p_{t}+1\right) \omega\right]$. Obviously, $p_{t} \rightarrow \infty$ as $t \rightarrow \infty$. From the third equation of system (1) we have

$$
\begin{aligned}
y(t)= & y\left(t_{1}+h\right) \\
& \times \exp \int_{t_{1}+h}^{t}\left[-g(s)+h(s) \int_{-h}^{0} k_{21}(\mu) \phi\right. \\
& \left.\times\left(x_{1}(\mu+s)\right) d \mu\right] d s \\
\leq & y\left(t_{1}+h\right) \exp \int_{t_{1}+h}^{t}\left[-g(s)+h(s) \phi\left(\epsilon_{0}\right)\right] d s \\
\leq & y\left(t_{1}+h\right) \exp \left\{\int_{t_{1}+h}^{t_{1}+h+p_{t} \omega}+\int_{t_{1}+h+p_{t} \omega}^{t}\right\} \\
& \times\left[-g(s)+h(s) \phi\left(\epsilon_{0}\right)\right] d s \\
\leq & y\left(t_{1}+h\right) \exp \left(r^{*} \omega\right) \\
& \times \exp \left\{p_{t} \int_{0}^{\omega}\left[-g(s)+h(s) \phi\left(\epsilon_{0}\right)\right] d s\right\},
\end{aligned}
$$

where $r^{*}=\max _{0 \leq t \leq+\infty}\left\{|g(t)|+h(t) \phi\left(\epsilon_{0}\right)\right\}$. Hence, from (25), we obtain $y(t) \rightarrow 0$ as $t \rightarrow \infty$. This leads to a contradiction. Thus, (26) holds.

Now, we prove that (23) is true. Otherwise, there is a sequence of initial functions $\left\{\psi_{n}\right\} \subset C_{+}$for system (1) such that

$$
\limsup _{t \rightarrow \infty} y\left(t, \psi_{n}\right)>\left(M_{0}+1\right) n, \quad \forall n=1,2,3, \ldots
$$

In view of (26), for each $n$, there are time sequences $\left\{s_{q}^{(n)}\right\}$ and $\left\{t_{q}^{(n)}\right\}$, satisfying $0<s_{1}^{(n)}<t_{1}^{(n)}<s_{2}^{(n)}<t_{2}^{(n)}<\cdots<s_{q}^{(n)}<$ $t_{q}^{(n)}<\cdots$ and $s_{q}^{(n)} \rightarrow \infty$ as $q \rightarrow \infty$, such that

$$
\begin{aligned}
& y\left(s_{q}^{(n)}, \psi_{n}\right)=M_{0}, \quad y\left(t_{q}^{(n)}, \psi_{n}\right)=\left(M_{0}+1\right) n, \\
& M_{0}<y\left(t, \psi_{n}\right)<\left(M_{0}+1\right) n, \quad \forall t \in\left(s_{q}^{(n)}, t_{q}^{(n)}\right) .
\end{aligned}
$$

By the ultimate boundedness of $\left(x_{1}\left(t, \psi_{n}\right), x_{2}\left(t, \psi_{n}\right)\right)$, for each $n$, there is a constant $T^{(n)}>0$ such that $x_{1}\left(t, \psi_{n}\right)<M_{1}$ for 
all $t \geq T^{(n)}$. Further, for each $n$ there is $K^{(n)}>0$ such that $s_{q}^{(n)}>T^{(n)}+h$ for all $q \geq K^{(n)}$. Hence, for all $q \geq K^{(n)}$, directly from system (1) we have

$$
\begin{aligned}
y\left(t_{q}^{(n)}, \psi_{n}\right)= & y\left(s_{q}^{(n)}, \psi_{n}\right) \\
& \times \exp \int_{s_{q}^{(n)}}^{t_{q}^{(n)}}\left[-g(s)+h(s) \int_{-h}^{0} k_{21}(\mu) \phi\right. \\
& \left.\times\left(x_{1}\left(\mu+s, \psi_{n}\right)\right) d \mu\right] d s \\
\leq & y\left(s_{q}^{(n)}, \psi_{n}\right) \exp \int_{s_{q}^{(n)}}^{t_{q}^{(n)}}\left[-g(s)+h(s) \phi\left(M_{1}\right)\right] d s \\
\leq & y\left(s_{q}^{(n)}, \psi_{n}\right) \exp \left[r_{1}\left(t_{q}^{(n)}-s_{q}^{(n)}\right)\right],
\end{aligned}
$$

where $r_{1}=\max _{t \in[0,+\infty)}\left\{|g(t)|+h(t) \phi\left(M_{1}\right)\right\}$. Consequently, by (32) we have

$$
t_{q}^{(n)}-s_{q}^{(n)} \geq \frac{\ln n}{r_{1}}, \quad \forall q \geq K^{(n)}
$$

Hence, for any constant $L>0$, there is a $N_{0}>0$ such that $t_{q}^{(n)}>s_{q}^{(n)}+2 L+h$ for all $n \geq N_{0}$ and $q \geq K^{(n)}$. For any fixed $n \geq N_{0}$ and $q \geq K^{(n)}$, we prove that there must be $\tilde{t}_{1} \in$ $\left[s_{q}^{(n)}+h, s_{q}^{(n)}+L+h\right]$ such that $x_{1}\left(\widetilde{t}_{1}, \psi_{n}\right)<\epsilon_{0}$. Otherwise, if $x_{1}\left(t, \psi_{n}\right) \geq \epsilon_{0}$ for all $t \in\left[s_{q}^{(n)}+h, s_{q}^{(n)}+L+h\right]$, then, directly from system (1), we have by (24) and (33). Consider

$$
\begin{aligned}
\frac{d x_{1}\left(t, \psi_{n}\right)}{d t} \leq & a(t) x_{2}\left(t, \psi_{n}\right) \\
& -p(t) \phi\left(x_{1}\left(t, \psi_{n}\right)\right) \int_{-h}^{0} k_{12}(s) y\left(t+s, \psi_{n}\right) d s \\
\leq & a(t) x_{2}\left(t, \psi_{n}\right)-p(t) \phi\left(x_{1}\left(t, \psi_{n}\right)\right) M_{0} \\
< & M_{1} a^{m}-p^{l} M_{0} \phi\left(\epsilon_{0}\right)<-\epsilon_{0} .
\end{aligned}
$$

We can choose enough large $L>0$ such that $M_{1}-L \epsilon_{0}<0$. Integrating this inequality from $s_{q}^{(n)}+h$ to $s_{q}^{(n)}+L+h$, we have

$$
\begin{aligned}
x_{1}\left(s_{q}^{(n)}+L+h, \psi_{n}\right)< & x_{1}\left(s_{q}^{(n)}+h, \psi_{n}\right) \\
& -\epsilon_{0} L \leq M_{1}-L \epsilon_{0}<0 .
\end{aligned}
$$

This leads to a contradiction. Next, we prove $x_{1}\left(t, \psi_{n}\right)<\epsilon_{0}$ for all $t \in\left[\tilde{t}_{1}, t_{q}^{(n)}\right]$. Otherwise, there is a $\widetilde{t}_{2}>\widetilde{t}_{1}$ such that $x_{1}\left(\tilde{t}_{2}, \psi_{n}\right)=\epsilon_{0}$ and $x_{1}\left(t, \psi_{n}\right)<\epsilon_{0}$ for all $t \in\left(\tilde{t}_{1}, \tilde{t}_{2}\right)$. Then, we have $d x_{1}\left(\widetilde{t}_{2}, \psi_{n}\right) / d t \geq 0$. On the other hand, a similar calculation as in (36), we have

$$
\frac{d x_{1}\left(\tilde{t}_{2}, \psi_{n}\right)}{d t} \leq M_{1} a^{m}-p^{l} M_{0} \phi\left(\epsilon_{0}\right)<-\epsilon_{0} .
$$

This leads to a contradiction. Therefore, $x_{1}(t)<\epsilon_{0}$ for all $t \epsilon$ $\left[s_{q}^{(n)}+L+h, t_{q}^{(n)}\right]$ for all $n \geq N_{0}$ and $q \geq K^{(n)}$. From (32) and (33), we have

$$
\begin{aligned}
\left(M_{0}+1\right) n= & y\left(t_{q}^{(n)}, \psi_{n}\right)=y\left(s_{q}^{(n)}+L+h, \psi_{n}\right) \\
\times & \times \exp \int_{s_{q}^{(n)}+L+h}^{t_{q}^{(n)}}\left[-g(t)+h(t) \int_{-h}^{0} k_{21}\right. \\
& \left.\times(s) \phi\left(x_{1}\left(t+s, \psi_{n}\right)\right) d s\right] d t \\
\leq & y\left(s_{q}^{(n)}+L+h, \psi_{n}\right) \\
& \times \exp \int_{s_{q}^{(n)}+L+h}^{t_{q}^{(n)}}\left[-g(t)+h(t) \phi\left(\epsilon_{0}\right)\right] d t \\
< & \left(M_{0}+1\right) n \exp \int_{s_{q}^{(n)}+L+h}^{t_{q}^{(n)}}[-g(t) \\
& \left.+h(t) \phi\left(\epsilon_{0}\right)\right] d t .
\end{aligned}
$$

From (25) we can choose large enough constant $L>0$ such that

$$
\exp \int_{t}^{t+L}\left[-g(t)+h(t) \phi\left(\epsilon_{0}\right)\right] d t<1
$$

for all $t \in R_{+}$. Hence, from (39) we finally obtain a contradiction $\left(M_{0}+1\right) n<\left(M_{0}+1\right) n$. This shows that (23) holds. Choose a constant $M=\max \left\{M_{1}, M_{2}\right\}$. Then we obtain that the conclusion of Theorem 7 is true. This completes the proof.

Proof of Theorem 9. We will use Lemma 6 to prove this theorem. We choose space

$$
\begin{aligned}
X=\{\psi & =\left(\psi_{1}, \psi_{2}, \psi_{3}\right): \psi_{i}(\theta)>0, \\
& \left.i=1,2, \psi_{3}(\theta) \geq 0, \forall \theta \in[-h, 0]\right\},
\end{aligned}
$$

and sets $X^{0}$ and $\partial X^{0}$ are defined by

$$
\begin{gathered}
X^{0}=\left\{\psi=\left(\psi_{1}, \psi_{2}, \psi_{3}\right) \in X: \psi_{3}(0)>0\right\}, \\
\partial X^{0}=\left\{\psi=\left(\psi_{1}, \psi_{2}, \psi_{3}\right) \in X: \psi_{3}(\theta) \equiv 0, \forall \theta \in[-h, 0]\right\} .
\end{gathered}
$$

For any $\psi \in X$, let $x(t, \psi)=\left(x_{1}(t, \psi), x_{2}(t, \psi), y(t, \psi)\right)$ be the solution of system (1) with initial value $\psi$ at $t=0$. We define continuous map $P$ in Lemma 6 as follows:

$$
P(\psi)=x_{\omega}(\psi), \quad \psi \in X,
$$

where $x_{\omega}(\psi)=x(\omega+s, \psi)$ with $s \in[-h, 0]$.

Now, we verify that all the conditions of Lemma 6 will be satisfied for map $P$. It is easy to see that $X^{0}$ and $\partial X^{0}$ are positively invariant. From the expression of right side functional $f(t, \phi)$ of system (1), we can directly obtain that, 
for any bounded set $A \subset X$, there is a constant $M(A)>0$ such that $|f(t, \phi)| \leq M(A)$ for all $t \geq 0$ and $\phi \in A$. By the Ascoli-Arzela theorem, it implies that map $P$ is compact on $X$; that is, for any bounded set $B \subset X$, set $P(B)=\{P(\psi)=$ $\left.x_{\omega}(\psi): \psi \in B\right\}$ is precompact. Moreover, by Theorem 7, we obtain that map $P$ is also point dissipative on $X$.

Further, we define

$$
M_{\partial}=\left\{\psi \in \partial X^{0}: P^{m}(\psi) \in \partial X^{0}, \forall m>0\right\},
$$

where $P^{m}=P\left(P^{m-1}\right)$ for all $m>1$ and $P^{1}(\psi)=P(\psi)$. Obviously, we have $M_{\partial}=\partial X^{0}$.

Denote by $\omega(\psi)$ the $\omega$-limit set of solution $x(t, \psi)$ of system (1) starting at $t=0$ with initial value $\psi \in X$. Let

$$
\Omega\left(M_{\partial}\right)=\bigcup\left\{\omega(\psi): \psi \in M_{\partial}\right\} .
$$

From Remark 2, there is a fixed point of map $P$ in $M_{\partial}$, which is $M_{1}=\left(x_{1}^{*}(0), x_{2}^{*}(0), 0\right)$.

From (10), we can choose a constant $\epsilon_{0}>0$ such that

$$
A_{\omega}\left(-g(t)+h(t) \int_{-h}^{0} k_{21}(s) \phi\left(x_{1}^{*}(t+s)-\epsilon_{0}\right) d s\right)>0 .
$$

By the continuity of solutions with respect to the initial value, for the above given constant $\epsilon_{0}>0$, there exists $\delta_{0}>0$ such that for all $\psi \in X^{0}$ with $\left\|\psi-M_{1}\right\| \leq \delta_{0}$, it follows that

$$
\left\|x_{t}(\psi)-x_{t}\left(M_{1}\right)\right\|<\epsilon_{0}, \quad \forall t \in[0, \omega] .
$$

Now, we prove

$$
\limsup _{t \rightarrow \infty} d\left(P^{m}(\psi), M_{1}\right) \geqslant \delta_{0} .
$$

Suppose the conclusion is not true, then

$$
\limsup _{t \rightarrow \infty} d\left(P^{m}(\psi), M_{1}\right)<\delta_{0}
$$

for some $\psi \in X^{0}$. Without loss of generality, we can assume that

$$
d\left(P^{m}(\psi), M_{1}\right)<\delta_{0}, \quad \forall m \geq 0 .
$$

Further, from (47) we have

$$
\left\|x_{t}\left(P^{m}(\psi)\right)-x_{t}\left(M_{1}\right)\right\|<\epsilon_{0}, \quad \forall m \geq 0, t \in[0, \omega] .
$$

For any $t \geq 0$, let $t=m \omega+t^{\prime}$, where $t^{\prime} \in[0, \omega]$ and $m=[t / \omega]$ are the greatest integers less than or equal to $[t / \omega]$, then we can get

$$
\begin{array}{r}
\left\|x_{t}(\psi)-x_{t}\left(M_{1}\right)\right\|=\left\|x_{t^{\prime}}\left(P^{m}(\psi)\right)-x_{t^{\prime}}\left(M_{1}\right)\right\|<\epsilon_{0}, \\
\forall t \geq 0 .
\end{array}
$$

Since $x_{t}(\psi)=\left(x_{1}(t+s, \psi), x_{2}(t+s, \psi), y(t+s, \psi)\right)$, and $x_{t}\left(M_{1}\right)=\left(x_{1}^{*}(t+s), x_{2}^{*}(t+s), 0\right)$ for all $s \in[-h, 0]$, it follows from (52) that, for all $t \geq-h$,

$$
\begin{gathered}
0 \leq y(t, \psi)<\epsilon_{0}, \quad\left|x_{1}(t, \psi)-x_{1}^{*}(t)\right|<\epsilon_{0}, \\
\left|x_{2}(t, \psi)-x_{2}^{*}(t)\right|<\epsilon_{0} .
\end{gathered}
$$

Then, by the third equation of system (1), we get, for any $t \geq 0$,

$$
\begin{aligned}
\frac{d y(t, \psi)}{d t}= & y(t, \psi) \\
& \times\left(-g(t)+h(t) \int_{-h}^{0} k_{21}(s) \phi\left(x_{1}(t+s, \psi)\right) d s\right) \\
\geq & y(t, \psi) \\
& \times(-g(t) \\
& \left.\quad+h(t) \int_{-h}^{0} k_{21}(s) \phi\left(x_{1}^{*}(t+s)-\epsilon_{0}\right) d s\right) .
\end{aligned}
$$

Therefore, we further have, for any $t \geq 0$,

$$
\begin{aligned}
y(t, \psi) \geq \psi(0) \exp \left(\int_{0}^{t}(\right. & -g(u)+h(u) \int_{-h}^{0} k_{21}(s) \phi \\
& \left.\left.\times\left(x_{1}^{*}(u+s)-\epsilon_{0}\right) d s\right) d u\right) .
\end{aligned}
$$

From (46) we can directly obtain that $\lim _{t \rightarrow \infty} y(t, \psi)=\infty$, which leads to a contradiction. Therefore, claim (48) holds. This shows that

$$
W^{s}\left(M_{1}\right) \cap X^{0}=\emptyset .
$$

From Lemma 1 we can obtain that $\left\{M_{1}\right\}$ is a global attractor of map $P$ in $M_{\partial}$; that is, each orbit of map $P$ in $M_{\partial}$ converges to $\left\{M_{1}\right\}$. Hence, $\left\{M_{1}\right\}$ is isolated in $M_{\partial}$, and, hence, in $X$ by (56). Furthermore, $\left\{M_{1}\right\}$ also is invariant and $\left\{M_{1}\right\}$ does not form a cycle in $M_{\partial}$ and, hence, in $\partial X^{0}$.

Therefore, all the conditions of Lemma 6 are satisfied. By Lemma 6 we finally obtain that map $P$ is uniformly persistent with respect to $\left(X^{0}, \partial X^{0}\right)$. Further, from Theorem 3.1.1 given in [10], we can obtain that all positive solutions of system (1) are uniformly persistent. This completes the proof.

Proof of Theorem 12. From (12), we can choose a constant $0<$ $\epsilon_{0}<1$, such that

$$
\begin{aligned}
& A_{\omega}\left[-g(t)+h(t) \int_{-h}^{0} k_{21}(s) \phi\left(x_{1}^{*}(t+s)\right) d s+h(t) \epsilon_{0}\right] \\
& \quad \leq-\epsilon_{0} .
\end{aligned}
$$

We first show that for any positive solution $\left(x_{1}(t), x_{2}(t), y(t)\right)$ of system (1) $\lim _{t \rightarrow \infty} y(t)=0$. Since

$$
\begin{aligned}
& \frac{d x_{1}(t)}{d t}<a(t) x_{2}(t)-b(t) x_{1}(t)-d(t) x_{1}^{2}(t), \\
& \frac{d x_{2}(t)}{d t}=c(t) x_{1}(t)-f(t) x_{2}^{2}(t),
\end{aligned}
$$


for all $t>0$. By the comparison theorem and Corollary 3, we obtain that, for any $\epsilon \in\left(0, \epsilon_{0}\right)$, there is a $T_{\epsilon}>0$ such that

$$
x_{i}(t)<x_{i}^{*}(t)+\epsilon, \quad \forall t \geq T_{\epsilon}, \quad i=1,2 .
$$

For any $t>T_{\epsilon}+h$, from system (1), we have

$$
\begin{aligned}
\frac{d y(t)}{d t}=y(t)\left[-g(t)+h(t) \int_{-h}^{0} k_{21}(s) \phi\left(x_{1}(t+s)\right) d s\right] \\
\leq y(t)[-g(t) \\
\left.+h(t) \int_{-h}^{0} k_{21}(s) \phi\left(x_{1}^{*}(t+s)+\epsilon_{0}\right) d s\right] .
\end{aligned}
$$

From (57) and (60), we obtain that $\lim _{t \rightarrow \infty} y(t)=0$.

Consider the following system with a parameter $\alpha$ :

$$
\begin{gathered}
\frac{d x_{1}(t)}{d t}=a(t) x_{2}(t)-b(t) x_{1}(t) \\
-d(t) x_{1}^{2}(t)-\alpha p(t) x_{1}(t), \\
\frac{d x_{2}(t)}{d t}=c(t) x_{1}(t)-f(t) x_{2}^{2}(t) .
\end{gathered}
$$

From Lemma 1 we obtain that (61) has a unique globally asymptotically stable positive $\omega$-periodic solution $\left(x_{1 \alpha}^{*}(t), x_{2 \omega}^{*}(t)\right)$. By the continuity of solutions with respect to the parameter, we further obtain

$$
\lim _{\alpha \rightarrow 0}\left(x_{1 \alpha}^{*}(t), x_{2 \omega}^{*}(t)\right)=\left(x_{1}^{*}(t), x_{2}^{*}(t)\right),
$$

where $\left(x_{1}^{*}(t), x_{2}^{*}(t)\right)$ is the globally asymptotically stable positive $\omega$-periodic solution of system (6). Therefore, for any $\epsilon>0$ there is an $\alpha_{0}>0$ such that, for all $t \in R_{+}$,

$$
x_{i \alpha_{0}}^{*}(t)>x_{i}^{*}(t)-\frac{\epsilon}{2}, \quad i=1,2 .
$$

Let $\bar{\phi}=\sup \left\{\phi\left(x_{1}\right) / x_{1}: 0 \leq x_{1} \leq M_{1}\right\}$, then, from assumption $\left(A_{3}\right)$, we obtain $0<\bar{\phi}<\infty$, where $M_{1}$ is given in Theorem 7. Since $\lim _{t \rightarrow \infty} y(t)=0$, there is a $T_{0}>0$ such that $y(t)<\alpha_{0} / \bar{\phi}$ for all $t \geq T_{0}$. Hence, for any $t \geq T_{0}+h$, we have

$$
\begin{aligned}
\frac{d x_{1}(t)}{d t}= & a(t) x_{2}(t)-b(t) x_{1}(t) \\
& -d(t) x_{1}^{2}(t)-\alpha_{0} p(t) x_{1}(t), \\
\frac{d x_{2}(t)}{d t} & =c(t) x_{1}(t)-f(t) x_{2}^{2}(t) .
\end{aligned}
$$

From the comparison theorem and Lemma 1, we can obtain that there is a $T_{1}>T_{0}$ such that, for all $t \geq T_{1}$,

$$
x_{i}(t)>x_{i \alpha_{0}}^{*}(t)-\frac{\epsilon}{2}>x_{i}^{*}(t)-\epsilon, \quad i=1,2 .
$$

Combining (59), we finally obtain that, for all $t \geq \max \left\{T_{\epsilon}, T_{1}\right\}$,

$$
x_{i}^{*}(t)-\epsilon<x_{i}(t)<x_{i}^{*}(t)+\epsilon, \quad i=1,2 .
$$

Therefore, $\lim _{t \rightarrow \infty} x_{i}(t)=x_{i}^{*}(t)(i=1,2)$. This completes the proof.

\section{Examples and Numerical Simulations}

In order to testify the validity of results, we consider the following predator-prey system. The system was obtained by letting $k_{12}(s)=\delta\left(s+\tau_{1}\right)$ and $k_{21}(s)=\delta\left(s+\tau_{2}\right)$ in system $(1)$. Consider

$$
\begin{aligned}
& \frac{d x_{1}(t)}{d t}=a(t) x_{2}(t)-b(t) x_{1}(t) \\
& \quad-d(t) x_{1}^{2}(t)-p(t) \phi\left(x_{1}(t)\right) y\left(t-\tau_{1}\right), \\
& \frac{d x_{2}(t)}{d t}=c(t) x_{1}(t)-f(t) x_{2}^{2}(t), \\
& \frac{d y(t)}{d t}=y(t)\left[-g(t)+h(t) \phi\left(x_{1}\left(t-\tau_{2}\right)\right)\right] .
\end{aligned}
$$

The corresponding prey subsystem is

$$
\begin{aligned}
& \frac{d x_{1}(t)}{d t}=a(t) x_{2}(t)-b(t) x_{1}(t)-d(t) x_{1}^{2}(t), \\
& \frac{d x_{2}(t)}{d t}=c(t) x_{1}(t)-f(t) x_{2}^{2}(t) .
\end{aligned}
$$

Example 1. In system (67), we let $a(t)=1.2+\sin (2 \pi t)$, $b(t)=0.2, d(t)=0.2, c(t)=0.5 \sin (2 \pi t)+0.75, f(t)=$ $0.8, p(t)=0.4, g(t)=0.28+0.1 \sin (\pi t / 2), h(t)=2.1+$ $2 \sin (\pi t / 3), \tau_{1}=0.2, \tau_{2}=0.3$, and $\phi\left(t, x_{1}(t)\right)=\left(x_{1}(t)\right) /(9+$ $\left.x_{1}^{2}(t)\right)$. We take different initial functions $\psi=\left(\psi_{1}, \psi_{2}, \psi_{3}\right)=$ $(2.5+k, 1+k, 0.3+k), k=0,1,2,3,4,5$ for all $s \in$ $[-0.3,0]$. We easily verify that assumptions $\left(A_{1}\right)-\left(A_{3}\right)$ hold. Therefore, from Lemma 1, system (68) has a unique globally asymptotically stable positive periodic solution $\left(x_{1}^{*}(t), x_{2}^{*}(t)\right)$. By the numerical simulations, we get that the upper and lower bounds of periodic function $x_{1}^{*}(t)$ are 2.87 and 1 , respectively.

It is easy to verify that condition (10) in Theorem 9 also holds. Therefore, from Theorem 9 and Corollary 14, we obtain that system (67) is ultimately bounded and permanent and at least has a positive periodic solution. The numerical simulations of the above results can be seen in Figures 1, 2, 3, and 4 .

Remark 1. There is an open question: from Figure 3, we see that $y(t)$ of system (1) has more than one periodic solution. So, we cannot get a globally asymptotically stability solution of system (1). Whether we can get a globally asymptotically stability solution of system (1) under some conditions is our future work.

Example 2. In system (67), the coefficients $g(t), h(t), p(t), \tau_{1}$, $\tau_{2}$, and $\phi\left(t, x_{1}(t)\right)$ are given as in Example 1. But, the other coefficients in system (67) are given as the following different values: $a(t)=1+\sin (2 \pi t), c(t)=0.2 \sin (2 \pi t)+0.75$, $b(t)=0.25, d(t)=0.35$, and $f(t)=1$. We see that coefficients $a(t)$ and $c(t)$ are decreased and coefficients $b(t)$, $d(t)$, and $f(t)$ are increased. From Corollary 3, system (68) has a unique globally asymptotically stable positive periodic solution $\left(x_{1}^{*}(t), x_{2}^{*}(t)\right)$. Moreover, compared with Example 1, we easily see that $x_{1}^{*}(t)$ and $x_{2}^{*}(t)$ will decrease. Further, 


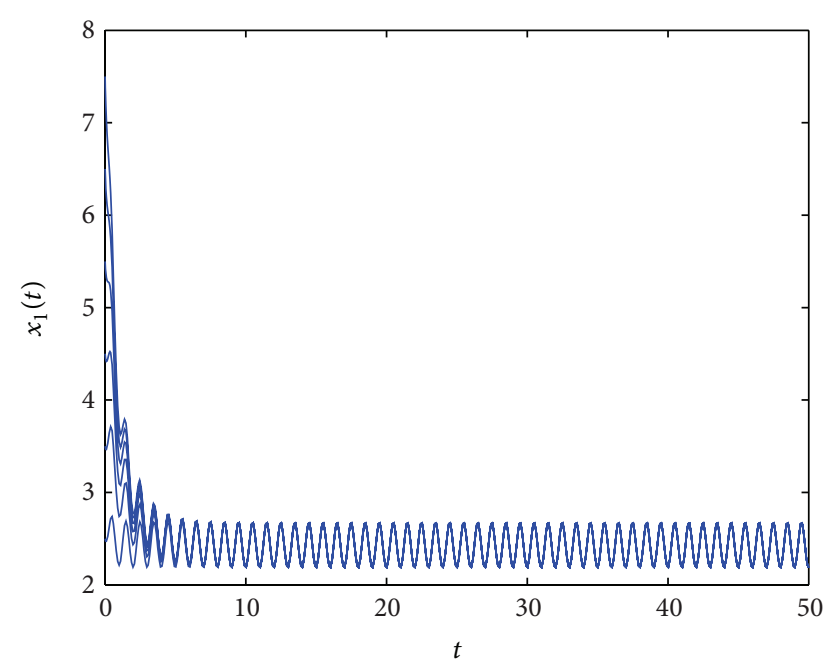

FIGURE 1

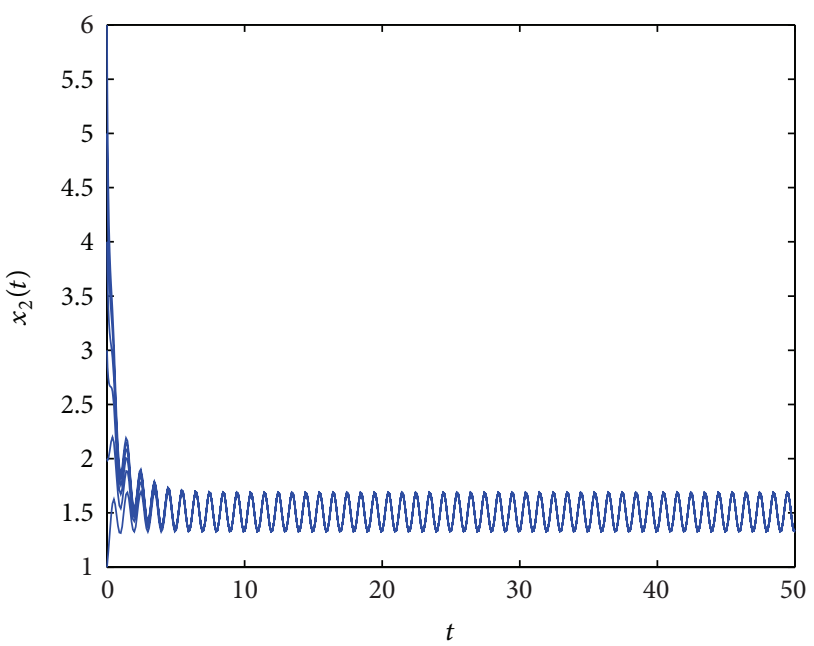

FIGURE 2

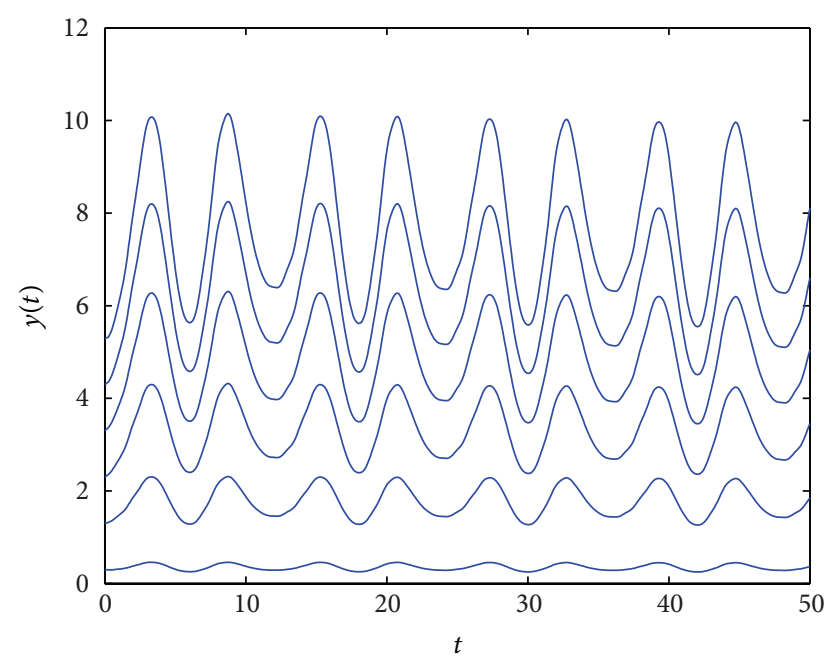

Figure 3

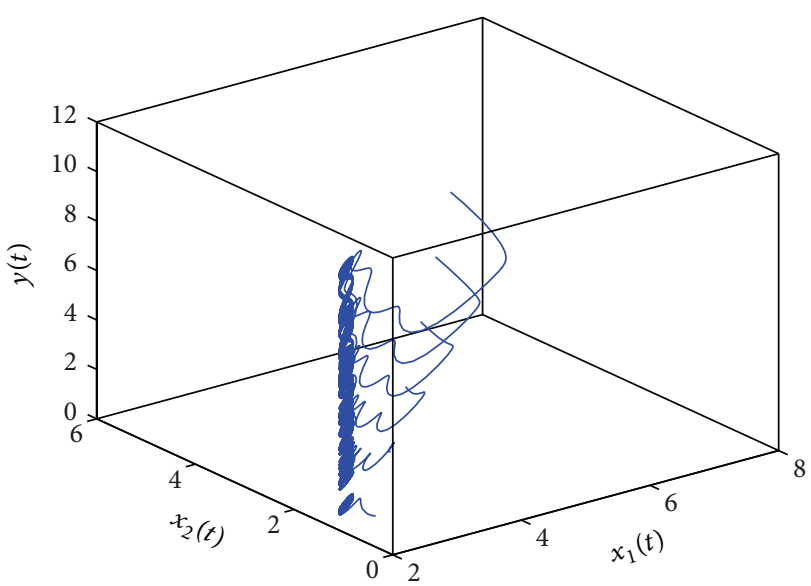

FIGURE 4

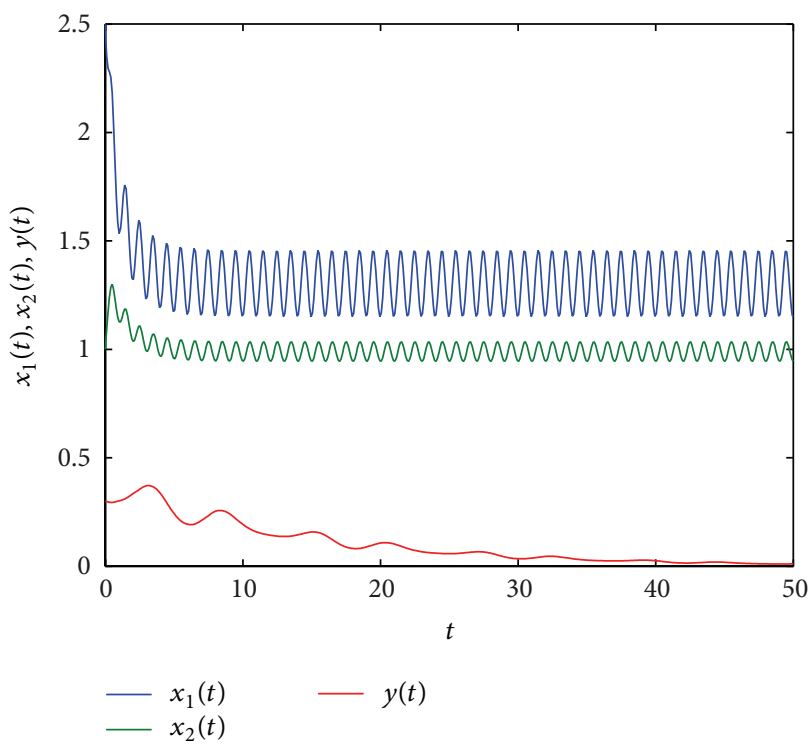

Figure 5

we easily verify that assumptions $\left(A_{1}\right)-\left(A_{3}\right)$ hold. It is easy to verify that condition (10) in Theorem 9 does not hold, but condition (12) in Theorem 12 holds. Therefore, from Theorem 12, we obtain that predator $y(t)$ in system (67) will become into extinction. The numerical simulations of the above results can be seen in Figures 5 and 6 by taking initial function $\psi=\left(\psi_{1}, \psi_{2}, \psi_{3}\right)=(2.5,1,5)$ for all $s \in[-0.3,0]$.

Remark 2. From the numerical simulations given in Examples 1 and 2, we see that the stage-structure in the prey, specially the birthrate, mortality, density restriction of infancy prey, the transformation from the infancy prey to the maturity prey, and the mortality and density restriction of maturity prey, will bring the very obvious effect for the permanence and extinction of the predator.

Example 3. In system (67), $a(t), b(t), d(t), c(t), f(t), p(t), \tau_{1}$, $\tau_{2}$, and $\phi\left(t, x_{1}(t)\right)$ are given as in Example 1, but $g(t)=0.28+$ $0.3 \sin (\pi t / 2)$ and $h(t)=2.1+2 \sin (\pi t / 7)$. We take initial 


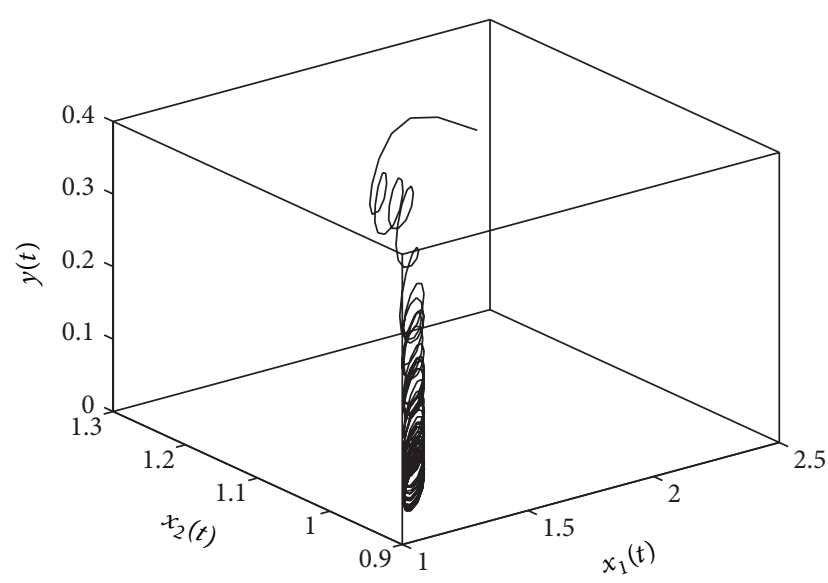

Figure 6

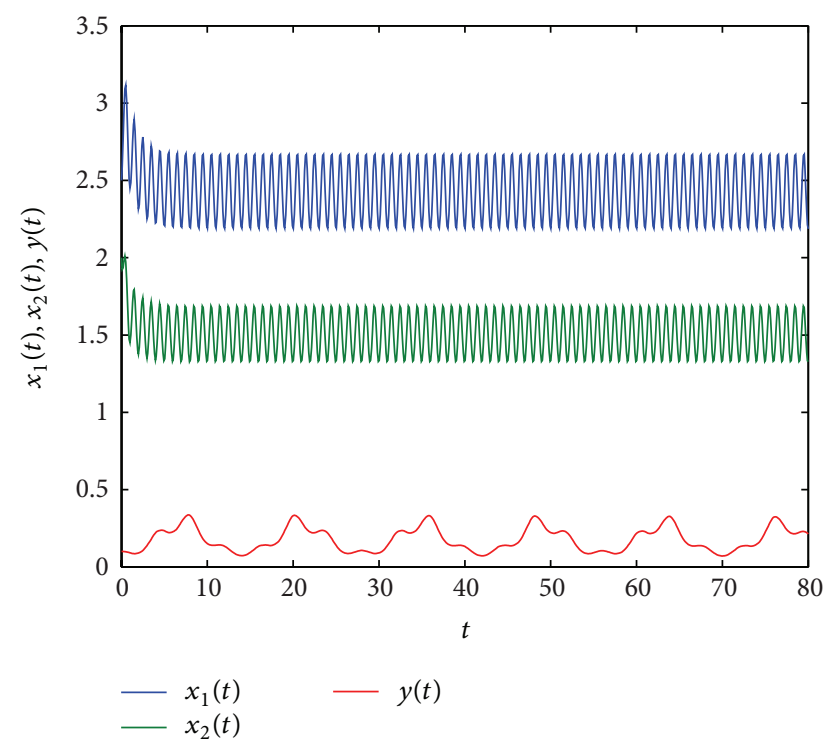

FIGURE 7

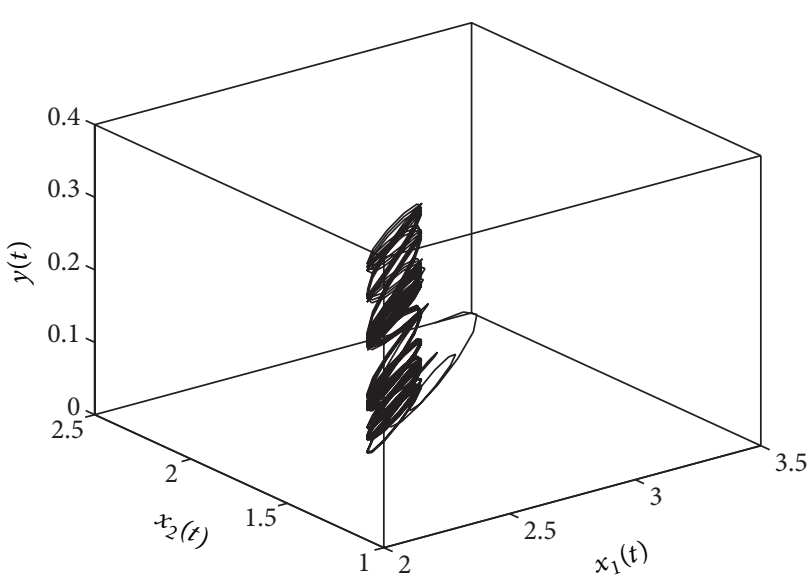

Figure 8

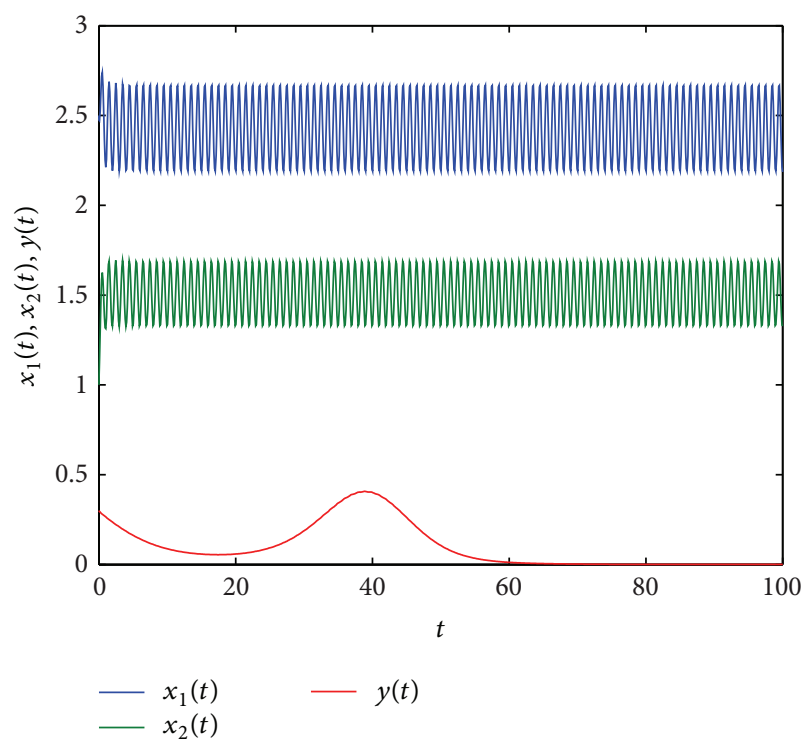

FIGURE 9 function $\psi=\left(\psi_{1}, \psi_{2}, \psi_{3}\right)=(2.5,2,0.1)$ for all $s \in[-0.3,0]$. We easily verify that assumptions $\left(A_{1}\right)-\left(A_{3}\right)$ hold. From Example 1, system (68) has a unique globally asymptotically stable positive periodic solution $\left(x_{1}^{*}(t), x_{2}^{*}(t)\right)$, and the upper and lower bounds of periodic function $x_{1}^{*}(t)$ are 2.87 and 1 , respectively.

It is easy to verify that condition (10) in Theorem 9 does not hold. Therefore, Theorem 9 and Corollary 14 are invalid. Numerical simulations of the above results can be seen in Figures 7 and 8 . From Figure 7, we see that the prey species $x$ is permanent; the predator species $y$ is permanent, too.

Example 4. In system (67), $a(t), b(t), d(t), c(t), f(t), p(t)$, $\tau_{1}, \tau_{2}$, and $\phi\left(t, x_{1}(t)\right)$ are given as in Example 1 , but $g(t)=$ $0.28+0.1 \sin (t /(2 \pi))$ and $h(t)=1.3+1.2 \sin (t /(5 \pi))$. We take initial function $\psi=\left(\psi_{1}, \psi_{2}, \psi_{3}\right)=(2.5,1,0.3)$ for all $s \in$ $[-0.3,0]$. We easily verify that assumptions $\left(A_{1}\right)-\left(A_{3}\right)$ hold. Therefore, from Lemma 1, system (68) has a unique globally asymptotically stable positive periodic solution $\left(x_{1}^{*}(t), x_{2}^{*}(t)\right)$.
By the numerical simulations, we get that the upper and lower bounds of periodic function $x_{1}^{*}(t)$ are 2.87 and 1 , respectively.

Take the upper bounds of periodic function $x_{1}^{*}(t)$ into condition (10), we easily verify that condition (10) in Theorem 9 will hold. But we obtain that predator $y(t)$ in system (67) is extinct. The numerical simulations of the above results can be seen in Figures 9 and 10. From Figure 9, we see that the prey species $x$ is permanent, while the predator species $y$ turns to extinction.

Example 5. In systems (67), $a(t), b(t), d(t), c(t), f(t), p(t)$, $\tau_{1}, \tau_{2}$, and $\phi\left(t, x_{1}(t)\right)$ are given as in Example 1, but $g(t)=$ $0.3+0.1 \sin (\pi t / 2)$ and $h(t)=2.3+0.5 \sin (\pi t / 7)$. We take initial function $\psi=\left(\psi_{1}, \psi_{2}, \psi_{3}\right)=(2.5,1,0.3)$ for all $s \in$ $[-0.3,0]$. We easily verify that assumptions $\left(A_{1}\right)-\left(A_{3}\right)$ hold. Therefore, from Lemma 1, system (68) has a unique globally asymptotically stable positive periodic solution $\left(x_{1}^{*}(t), x_{2}^{*}(t)\right)$. By the numerical simulations, we get that the upper and lower bounds of periodic function $x_{1}^{*}(t)$ are 2.87 and 1 , respectively. 


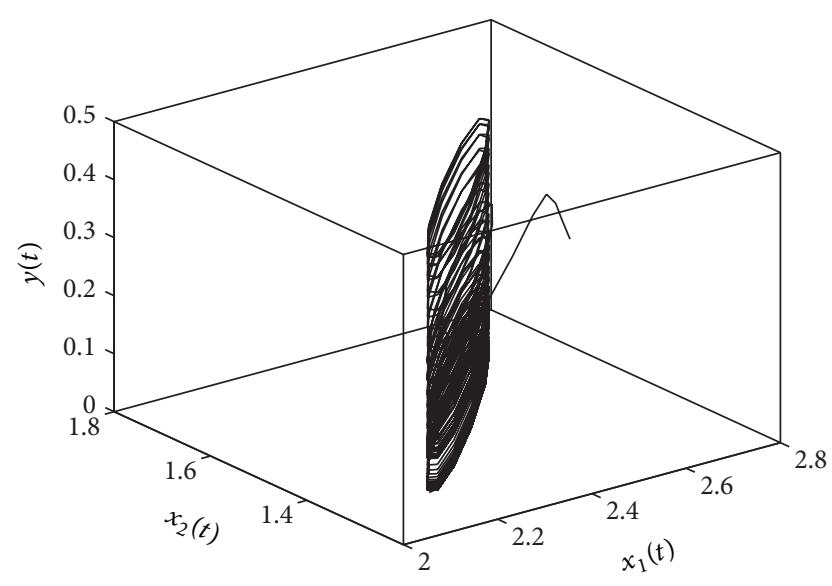

Figure 10

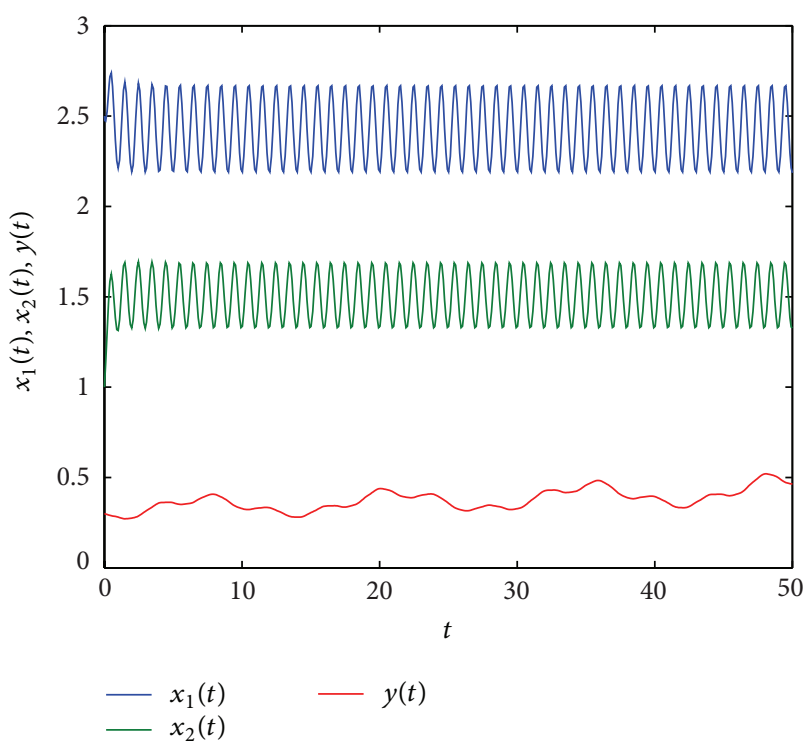

FIGURE 11

Take the lower bounds of periodic function $x_{1}^{*}(t)$ into condition (12), we easily verify that condition (12) in Theorem 12 will hold. But we obtain that predator $y(t)$ in system (67) is permanent. The numerical simulations of the above results can be seen in Figures 11 and 12. From Figure 12, we see that the prey species $x$ is permanent; the predator species $y$ is permanent, too.

\section{Conclusions}

In the real world, there are many types of interactions between two species. Predator-prey relations are among the most common ecological interactions.

In this paper, we study the global property in a delayed periodic predator-prey model with stage-structure in prey and density-independence in predator. The survival of species in a biological system is one of the most basic and important problems in mathematical biology, and permanence is an important concept when dealing with this problem. Here,

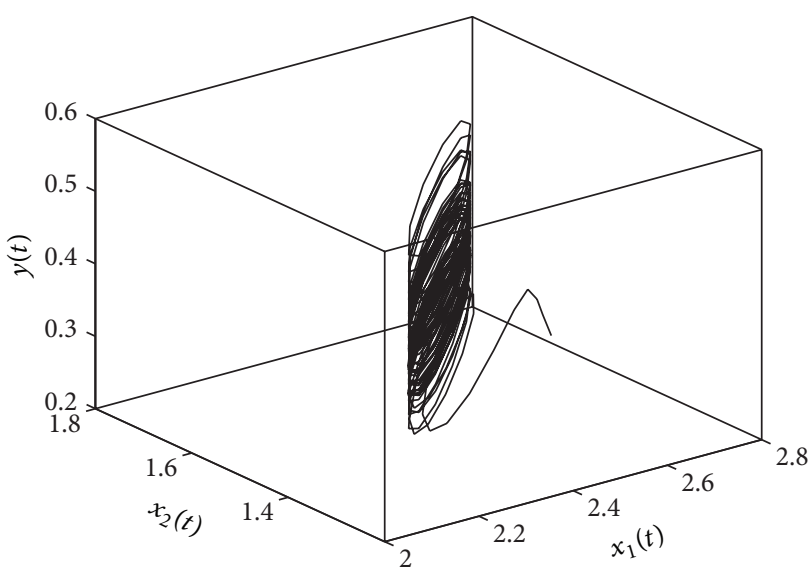

Figure 12

by using the analysis method, the comparison theorem of cooperative system, and the theory of the persistence of dynamical systems, we have established the integral form criteria on the ultimate boundedness, the sufficient integral conditions on the permanence and extinction of species. The method used in this paper is motivated by the works on the permanence and extinction for periodic predator-prey systems in patchy environment given by Teng and Chen in [5]. The results obtained in this paper are different from the predator-prey system given in [4], where the authors studied the necessary and sufficient integral conditions on permanence and extinction of species for nonautonomous predator-prey systems with infinite delays and predator density dependence. However, in our paper, we have considered the effects of general predator functional response on the survival of species. Therefore, we have modeled a general nonautonomous predator-prey system with finite delays and density independence. Some well-known results on the predator density-dependence are improved and extended to the predator density-independent cases.

\section{Conflict of Interests}

The authors declare that they have no financial and personal relationships with other people or organizations that can inappropriately influence their work; there is no professional or other personal interests of any nature or kind in any product, service, and/or company that could be construed as influencing the position presented in, or the review of, this paper.

\section{Acknowledgments}

This work is supported by the National Natural Science Foundation of China (Grant nos. 11271312, 11261056, 61164004, and 11301451), the China Postdoctoral Science Foundation (Grant nos. 20110491750 and 2012T50836), the Natural Science Foundation of Xinjiang (Grant nos. 2011211B08 and 2013211B06), and the Research Project at Xinjiang Institute of Engineering (2013XGZ281412). 


\section{References}

[1] W. Sokol and J. A. Howell, "Kinetics of phenol oxidation by washed cells," Biotechnology and Bioengineering, vol. 23, no. 9, pp. 2039-2049, 1980.

[2] J. Cui and X. Song, "Permanence of predator-prey system with stage structure," Discrete and Continuous Dynamical Systems B, vol. 4, no. 3, pp. 547-554, 2004.

[3] J. Cui and Y. Takeuchi, "A predator-prey system with a stage structure for the prey," Mathematical and Computer Modelling, vol. 44, no. 11-12, pp. 1126-1132, 2006.

[4] J. Cui and Y. Sun, "Permanence of predator-prey system with infinite delay," Electronic Journal of Differential Equations, vol. 2004, no. 81, pp. 1-12, 2004.

[5] Z. Teng and L. Chen, "Permanence and extinction of periodic predator-prey systems in a patchy environment with delay," Nonlinear Analysis: Real World Applications, vol. 4, no. 2, pp. 335-364, 2003.

[6] J. K. Hale, Theory of Functional Differential Equations, Springer, New York, NY, USA, 1977.

[7] J. K. Hale and J. Kato, "Phase space for retarded equations with infinite delay," Funkcialaj Ekvacioj, vol. 21, no. 1, pp. 11-41, 1978.

[8] Y. Kuang, Delay Differential Equation with Applications in Population Dynamics, Academic Press, New York, NY, USA, 1993.

[9] J. Cui, L. Chen, and W. Wang, "The effect of dispersal on population growth with stage-structure," Computers \& Mathematics with Applications, vol. 39, no. 1-2, pp. 91-102, 2000.

[10] X. Zhao, Dynamical Systems in Population Biology, Springer, New York, NY, USA, 2003.

[11] Z. Teng and L. Chen, "The positive periodic solutions of periodic Kolmogorov type systems with delays," Acta Mathematicae Applicatae Sinica, vol. 22, no. 3, pp. 446-454, 1999.

[12] H. L. Smith, "Cooperative systems of differential equations with concave nonlinearities," Nonlinear Analysis: Theory, Methods \& Applications, vol. 10, no. 10, pp. 1037-1052, 1986.

[13] X. Zhao, "The qualitative analysis of $n$-species Lotka-Volterra periodic competition systems," Mathematical and Computer Modelling, vol. 15, no. 11, pp. 3-8, 1991. 


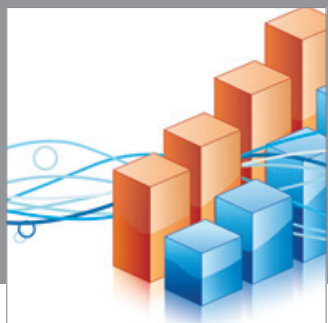

Advances in

Operations Research

mansans

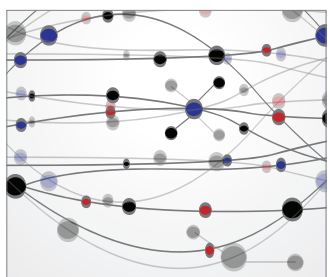

The Scientific World Journal
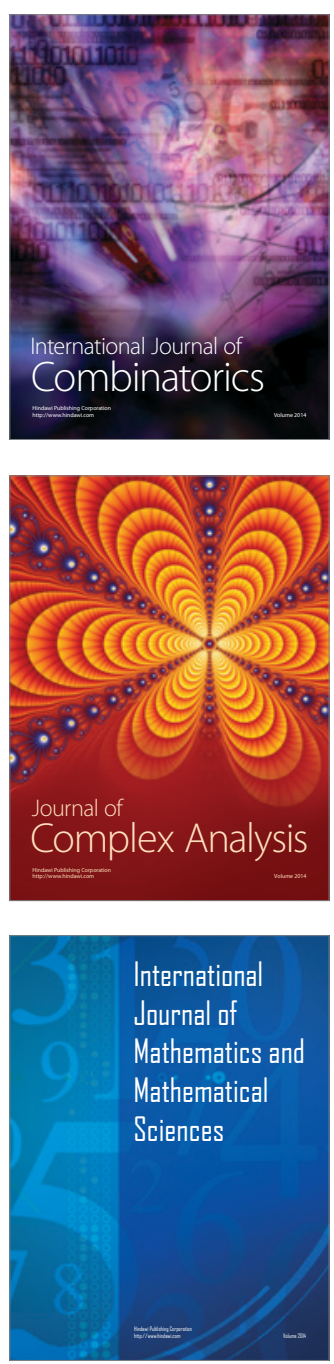
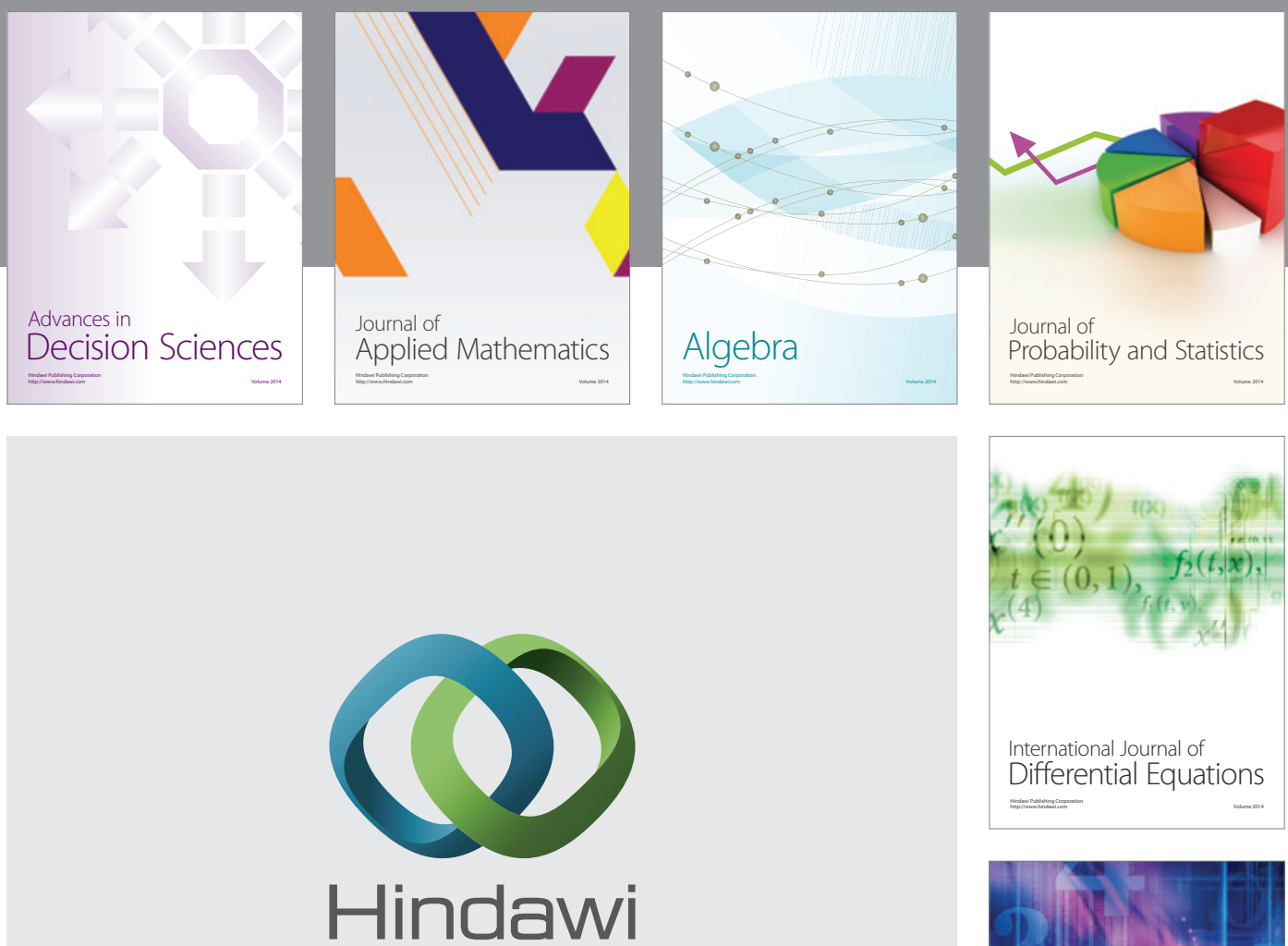

Submit your manuscripts at http://www.hindawi.com
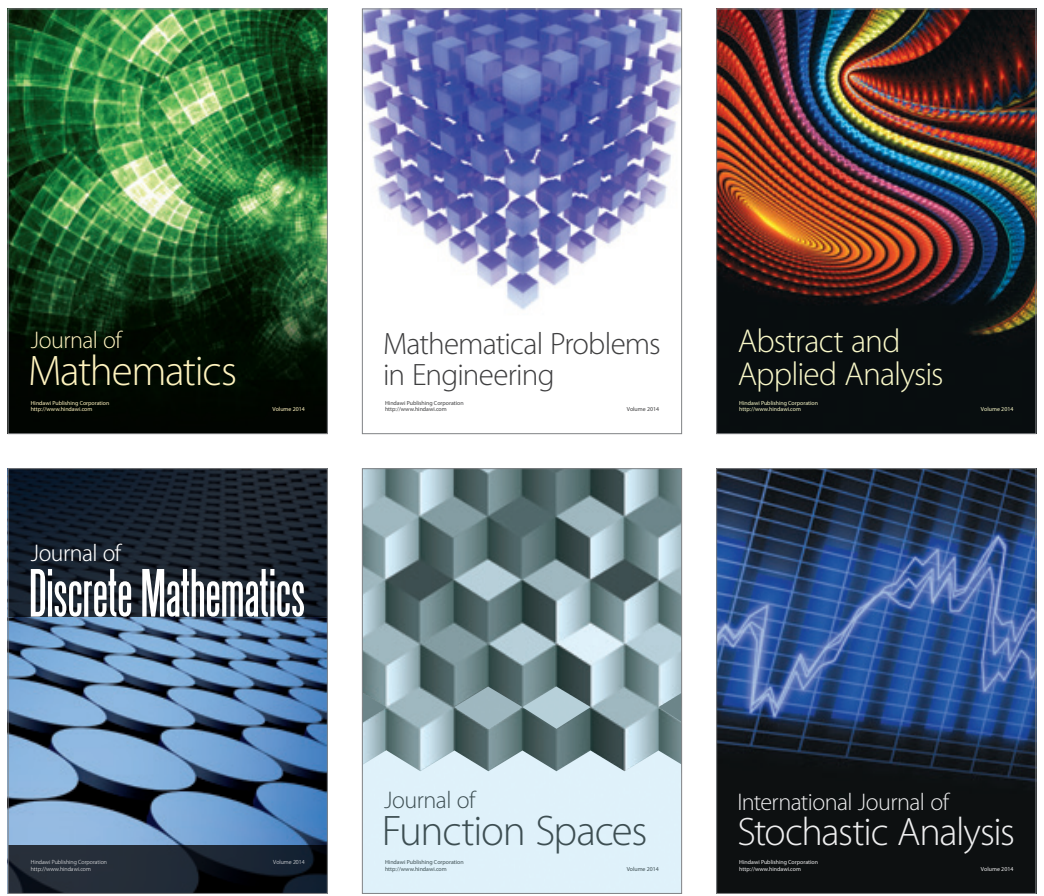

Journal of

Function Spaces

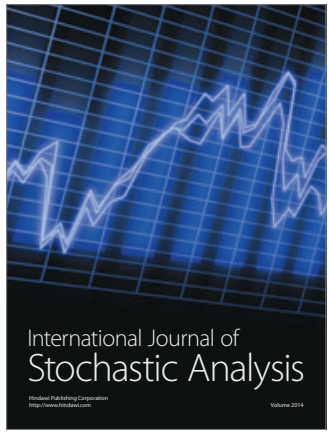

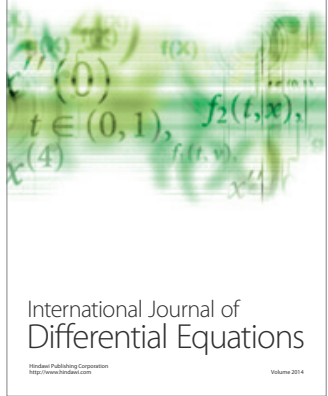
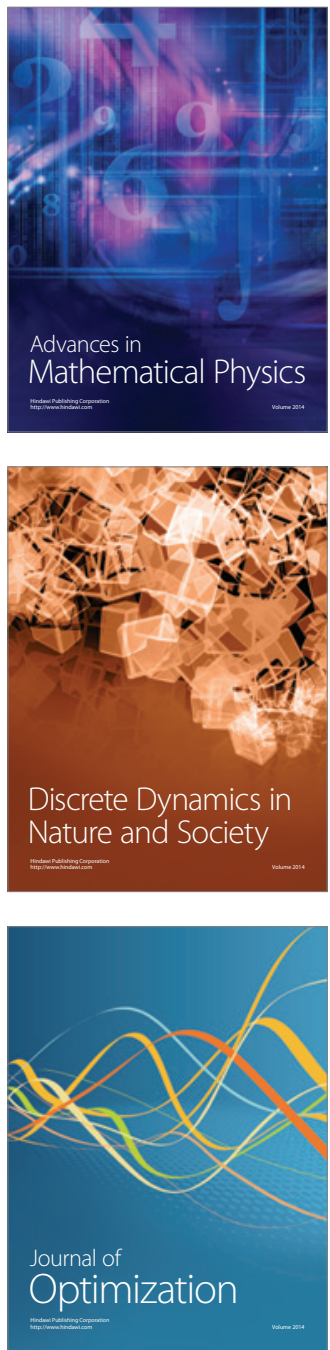